\title{
Gesundheit, Gebrechlichkeit und Komplexität
}

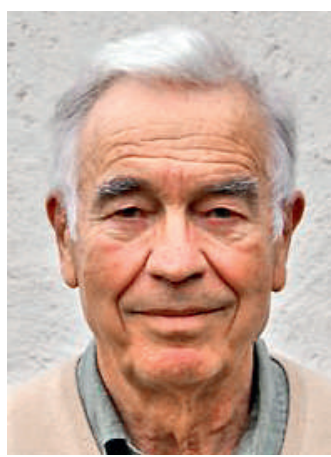

Hans Stalder
Im Jahr 1946 definierte die WHO Gesundheit als «Zustand des vollständigen körperlichen, geistigen und sozialen Wohlergehens und nicht nur das Fehlen von Krankheit oder Gebrechen» [1]. Diese Definition wurde seither nie verändert. Der grosse Fortschritt dieser Definition war, den psychischen und den sozialen Aspekt ebenso hoch zu gewichten wie den somatischen. Ich glaube, dass wir die Bedeutung des psychischen Aspekts begriffen haben: Mens sana in corpore sano. Wir haben gelernt, psychische Erkrankungen frühzeitig zu erkennen; die Zahl der Psychiater hat enorm zugenommen. Die Bedeutung des sozialen Aspekts hingegen scheint bisher nur vom öffentlichen Gesundheitswesen erfasst worden zu sein. Zuerst in England, dann in vielen anderen Ländern, darunter auch in der Schweiz, wurde nachgewiesen, dass soziale Faktoren im Gesundheitsbereich als Risikofaktoren für Morbidität und Mortalität eine wesentlich grössere für beide Definitionen - es sind unzählige - gilt: je mehr Kriterien, desto höher die Mortalität [2], was eigentlich nicht sehr überraschend ist ...

Wie soll man somit Gesundheit definieren, damit sie nicht nur ein unerreichbarer Idealzustand ist? Vor drei Jahren hat ein Expertengremium eine neue Definition vorgeschlagen: Danach ist Gesundheit «die Fähigkeit zur Anpassung und zum Selbstmanagement» [3]. Man müsste noch anfügen «auf körperlicher, psychischer und sozialer Ebene». Ich mag diesen Vorschlag, denn darin steckt die Idee, dass der Mensch ein komplexes System ist, zu dessen Hauptmerkmalen zählt, dass er sich auf neue Situationen einstellen kann, und dass ein früherer $\mathrm{Zu}$ stand (z.B. Gesundheit nach der Definition der WHO) nie wiederhergestellt werden kann. Man wäre demnach nur dann nicht gesund, wenn man sich nicht mehr anpassen kann, oder anders gesagt, wenn man seine Komplexität verloren hat. Somit

\section{«Wir tun uns schwer damit, eine Sozialanamnese zu erheben und sie in unsere klinischen Überlegungen einzubinden.»}

Rolle spielen als alle bekannten biologischen Risikofaktoren wie Bluthochdruck, Adipositas oder Cholesterin. Doch in der medizinischen Praxis haben wir kaum Fortschritte zu verzeichnen. Während sich unsere Kollegen Kardiologen und Lipidologen darüber streiten, ob niedrige LDL-C einen Zielwert darstellen oder die Werte nur gesenkt werden sollen, um unser Leben ein paar Wochen zu verlängern, tun wir uns immer noch schwer, eine Sozialanamnese $\mathrm{zu}$ erheben und sie in unsere klinischen Überlegungen einzubinden.

Das Problem der WHO-Definition ist natürlich, dass sie Gesundheit als Zustand vollkommenen Wohlergehens bezeichnet. Nach dieser Definition ist Gesundheit ein nicht erreichbares Ideal - vor allem dann, wenn wir Ärzte mit allen Mitteln nach einer Krankheit suchen und alles dafür tun, unseren Patienten ihre letzte Illusion von guter Gesundheit zu nehmen. Wir führen unzählige Tests durch, und wenn ein Wert nicht im Normbereich liegt, geben wir diesem Befund den Namen einer Krankheit z. B. Hypercholesterinämie -, die behandelt werden muss. Auch ältere Menschen werden davon nicht verschont - hierfür haben die Geriater den Begriff der «Frailty» (Gebrechlichkeit) erfunden. Er definiert sich entweder durch eine Kombination verschiedener rein körperlicher Beschwerden oder durch Hinzufügung sozialer und psychischer Parameter; könnte Gebrechlichkeit auch als Verlust der Komplexität definiert werden. Diese neue Definition für Gesundheit erklärt auch, warum so mancher, bei dem wir multiple Pathologien diagnostizieren, sich selbst als gesund bezeichnet: Er hat gelernt, sich anzupassen und mit seinen Beschwerden zu leben.

Die wichtigste Konsequenz dieses Gesundheitsbegriffs ist jedoch, dass unsere Tätigkeit weniger darin bestehen würde, mit allen möglichen Screenings, Tests und Gebrechlichkeitskriterien die Krankheit zu suchen, sondern vielmehr darin, die Anpassungs- und eigenständige Handlungsfähigkeit des Patienten wiederherzustellen, ihm also seine verlorene Komplexität wiederzugeben - und dies, vergessen wir es nicht, auf körperlicher, psychischer und sozialer Ebene.

Hans Stalder*

\section{Literatur}

1 Präambel der Verfassung der Weltgesundheitsorganisation, bei der Internationalen Gesundheitskonferenz in New York vom 19.-22. Juni 1946 verabschiedet und am 22. Juli 1946 von den Vertretern von 61 Staaten unterzeichnet. 1946 (Offizielle Dokumentation der Weltgesundheitsorganisation, Nr. 2, S. 100); in Kraft getreten am 7. April 1948.

2 Shamliyan T, Talley KM, Ramakrishnan R, Kane RL. Association of frailty with survival: a systematic literature review. Ageing Res Rev. 2013;719-36.

3 Huber M, Knottnerus JA, Green L, van der Horst H, Jadad AR, Kromhout D, et al. How should we define health? BMJ. 2011;343:235-7. 